7. Widmer AE, Dangel M. Alcohol-based handrub: evaluation of technique and microbiological efficacy with international infection control professionals. Infect Control Hosp Epidemiol 2004;25: 207-209.

\section{Response to McKinnell et al's Original Article "Cost-Benefit Analysis From the Hospital Perspective of Universal Active Screening Followed by Contact Precautions for Methicillin-Resistant Staphylococcus aureus Carriers"}

To the Editor-We read with interest the cost-benefit analysis by McKinnell et $\mathrm{al}^{1}$ who found that universal screening for methicillin-resistant Staphylococcus aureus (MRSA) may be relative costly for hospitals. We assessed the potential economic aspects of screening as part of a review of national MRSA control guidelines in Ireland. ${ }^{2}$ We found that MRSA screening is generally advocated as part of infection prevention and control measures, but an important consideration is the cost-effectiveness of the type of screening approach.

For patients admitted to acute hospitals setting, 7 studies (United States, 4; Germany, 1; United Kingdom, 1; Ireland, 1) compared the cost of universal screening with targeted screening of at-risk patients. Costs were limited to direct medical costs and were evaluated from the perspective of the healthcare provider or hospital. Four studies were cost comparisons, ${ }^{3-6} 2$ reported cost-effectiveness of the strategies compared with a base case of no screening and relative to each other, ${ }^{4,6}$ while 1 study provided a cost-benefit analysis of universal versus targeted screening. ${ }^{7}$ In hospitals where MRSA is endemic, screening (targeted or universal) reduced infection rates and was cost saving compared with a policy of no screening. ${ }^{3,4}$ Universal MRSA screening strategies were more effective but also more cost-intensive than targeted screening. ${ }^{4,6,7}$

In a retrospective review of a 3-year MRSA screening program that was implemented from 2006 to 2009 in the United Kingdom, only 7 extra MRSA cases were detected using universal screening compared with targeted screening, and in 1 month, universal screening generated 4,200 negative screens that incurred an additional $€ 25,488$ in laboratory costs. ${ }^{5}$ Similarly, a prospective study by Creamer et $\mathrm{al}^{8}$ found that extending screening to patients without risk factors (ie, universal screening) increased the number of screenings and the costs but did not result in the detection of a significant number of additional cases. In a 2011 US study, targeted screening was associated with lower costs and better outcomes than a policy of no screening, whereas universal screening was associated with an average cost-effectiveness ratio of $€ 11,769$ per MRSA infection. ${ }^{6}$ In a second cost-effectiveness analysis, targeted screening strategies were found to be more cost-effective than universal screening, with incremental cost-effectiveness ratios of $€ 3,227$ to $€ 28,507$, depending on the prevalence rate and testing used, compared with $€ 103,169$ to $€ 183,269$ per additional infection averted for universal screening. ${ }^{4}$ Finally, a US prospective study comparing the clinical effectiveness and cost benefit of universal versus targeted screening reported a benefit-to-cost ratio of 0.50 , indicating that for every additional euro spent on universal versus targeted screening, only $€ 0.40$ could be recovered in avoided costs due to a reduction in MRSA healthcareassociated infection. ${ }^{7}$

The control of MRSA is a multidisciplinary task involving surveillance, patient screening, decolonization, isolation and/ or the cohorting of patients, environmental decontamination, antimicrobial stewardship, maintenance of adequate staffing levels, and hand hygiene. Although considerable coordination efforts may need to be invested in control, we demonstrate that the evidence strongly suggests that overall MRSA prevention and control strategies are associated with significant cost savings. The control measures have additional merits because they increase the awareness of the importance of all healthcareassociated infections and their implementation decreases other healthcare-associated infections. ${ }^{9}$ However, MRSA control measures encompass a wide range of interventions, the efficacy and cost of some of which are dependent on prevalence rates, local resistance patterns, the characteristics of the patient population, and the hospital facilities, all of which will vary from country to country. Because the MRSA prevalence rate in Ireland is higher than in the United Kingdom and in other Northern European countries, MRSA prevention and control is very relevant in the potential efficient use of resources.

In conclusion, the evidence shows that screening, whether universal or targeted, is better than no screening, resulting in fewer MRSA infections. Although universal screening, as currently practiced in the United Kingdom, is the most costly but the most effective strategy, it is not as cost-effective as it is resource intensive. Universal screening detects few additional cases and results in a large number of additional negative screens. However, any evaluation of the effectiveness of screening methods should take account of healthcare costs, methods, the rapidity of test results, and the prevalence of colonization and infection.

\section{ACKNOWLEDGMENTS}

We are grateful to all members of the MRSA guideline development group for drafting and completing this set of national guidelines. We also acknowledge members of the National Clinical Effectiveness Committee and its Working Group for their work in the evaluation and quality assurance of the 2013 revised Irish national MRSA guidelines.

Financial support. This economic evaluation was undertaken by the National Clinical Effectiveness Committee (Department of Health, Ireland) with support from the Health Information and Quality Authority, Ireland. 
Potential conflicts of interest. H.H. reports that he has received research support from Steris, Pfizer (Ireland), and Cepheid and has received lecture and other fees from Novartis, AstraZeneca, Astellas, and Pall Medical. All other authors report no conflicts of interest relevant to this article.

\section{Mary Teresa O'Riordan, MRCPUK, MPH, MFPHMI; ${ }^{1}$ Patricia Harrington, $\mathrm{PhD}{ }^{2}$ Kathleen Mac Lellen, $\mathrm{PhD}{ }^{1}$ Máirín Ryan, $\mathrm{PhD} ;$; Hilary Humphreys, MD, FRCPI, FRCPath ${ }^{4,5}$}

\begin{abstract}
Affiliations: 1. Clinical Effectiveness Unit, Department of Health, Dublin, Ireland; 2. Health Technology Assessment Directorate, Health Information and Quality Authority, Dublin, Ireland; 3. Department of Pharmacology and Therapeutics, Trinity College Dublin, Ireland; 4. Department of Clinical Microbiology, Royal College of Surgeons in Ireland, Dublin, Ireland; 5. Department of Microbiology, Beaumont Hospital, Dublin, Ireland.

Address correspondence to Mary O'Riordan, MB, BAO, BcH, MRCPUK, Clinical Effectiveness Unit, Department of Health, Hawkins House, Hawkins Street, Dublin 2, Ireland (Mary_oriordan@health.gov.ie).

Infect Control Hosp Epidemiol 2015;36(7):856-857

(c) 2015 by The Society for Healthcare Epidemiology of America. All rights reserved. 0899-823X/2015/3607-0024. DOI: 10.1017/ice.2015.111
\end{abstract}

\section{REFERENCES}

1. McKinnell JA, Bartsch SM, Lee BY, Huang SS, Miller LG. Cost-benefit analysis from the hospital perspective of universal active screening followed by contact precautions for methicillinresistant Staphylococcus aureus carriers. Infect Control Hosp Epidemiol 2015;36:2-13.

2. National Clinical Effectiveness Committee. Prevention and control of methicillin-resistant Staphylococcus aureus (MRSA): National Clinical Guideline No. 2. 2013. Available at: http://health. gov.ie/wp-content/uploads/2015/01/National-Clinical-GuidelineNo.-2-MRSA1.pdf

3. Olchanski N, Mathews C, Fusfield L, Jarvis W. Assessment of the influence of test characteristics on the clinical and cost impacts of methicillin-resistant Staphylococcus aureus screening programs in US hospitals. Infect Control Hosp Epidemiol 2011;32: 250-257.

4. Tubbicke A, Hubner C, Flessa S. Cost comparison of MRSA screening and management-a decision tree analysis. BMC Health Serv Res 2012;12:438.

5. Collins J, Raza M, Ford M, Gould FK. Review of a three-year methicillin-resistant Staphylococcus aureus screening programme. J Hosp Infect 2011;78:81-85.

6. Kang J, Mandsager P, Biddle A, Weber D. Cost-effectiveness analysis of active surveillance screening for methicillin-resistant Staphylococcus aureus in an academic hospital setting. Infect Control Hosp Epidemiol 2012;33:477-486.

7. Leonhardt K, Yakusheva O, Costello M. Clinical effectiveness and cost benefit of universal versus targeted methicillin-resistant Staphylococcus aureus screening upon admission in hospitals. Infect Control Hosp Epidemiol 2011;32:797-803.

8. Creamer E, Galvin S, Humphreys H, et al. Evaluation of screening risk and nonrisk patients for methicillin-resistant Staphylococcus aureus on admission in an acute care hospital. Am J Infect Control 2012;40:411-415.
9. Rubinovitch B, Pittet D. Screening for methicillin-resistant Staphylococcus aureus in the endemic hospital: what have we learned? J Hosp Infect 2001;47:9-18.

\section{Reply to O'Riordan et al}

To the Editor-We appreciate the letter from O'Riordan et $\mathrm{al}^{1}$ in response to our article on the cost benefit of methicillinresistant Staphylococcus aureus (MRSA) screening followed by contact precautions in the hospital setting. ${ }^{2}$ We agree that MRSA screening can have an important role as part of infection and control measures. We would like to take the opportunity to highlight 2 important considerations related to cost and benefits of MRSA screening: (1) who is paying for the intervention and who realizes the benefit - that is, the economic perspective, and (2) what we are doing with the MRSA screening data, and particularly what is the resultant intervention efficacy.

Our analysis demonstrated that universal MRSA screening followed by contact precautions would reduce hospitalassociated MRSA infections but would result in costs to a hospital. Our findings of increased costs to the hospital remained robust, regardless of number of body sites tested or MRSA identification method. These results are consistent with the literature, including the excellent references presented by O'Riordan et $\mathrm{al},{ }^{1}$ and support the notion that hospital-wide, universal surveillance followed by contact precautions would incur significant costs to a single hospital.

Interestingly, if we look at how universal MRSA screening followed by contact precautions impacts the healthcare system as a whole, the program could result in cost savings. ${ }^{3,4}$ The fundamental dilemma is that the costs of hospital-based screening and isolation are borne by the individual hospital performing the screening, but the individual benefits of screening may be reaped only later or by external beneficiaries (eg, other hospitals or non-hospital-based care entities). We suggest that the payment and incentive structure in the US system should be changed to support the expenditures necessary for infection prevention programs to realize both local and regional benefit.

Another key finding from our study was that our results were sensitive to the efficacy of the MRSA intervention. Our intervention efficacy estimates were based on the assumption that MRSA screening results were used to apply contact precautions after a positive test result. We did not model a strategy of preemptive isolation or MRSA decolonization programs. ${ }^{5}$ Using a more efficacious intervention would have resulted in our model having lower costs for hospitals and potentially cost saving for the hospital.

We would like to highlight a recent analysis of the Randomized Evaluation of Decolonization versus Universal Clearance to Eliminate (REDUCE) MRSA trial that confirmed that a strategy 